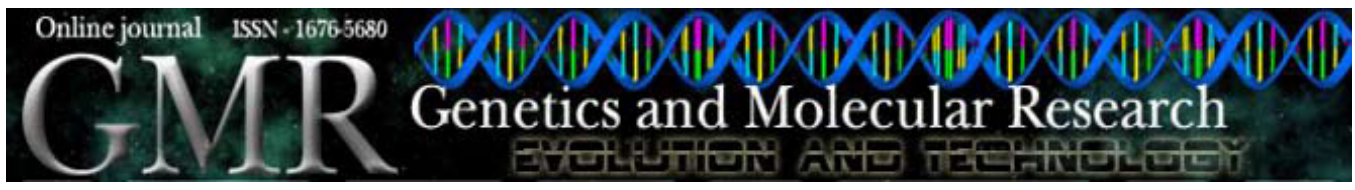

\title{
Study of the antimicrobial peptide indolicidin and a mutant in micelle medium by molecular dynamics simulation
}

C.A. Fuzo, J.R.M. Castro and L. Degrève

Grupo de Simulação Molecular, Faculdade de Filosofia, Ciências e Letras de Ribeirão Preto, Universidade de São Paulo, Ribeirão Preto, SP, Brasil

Corresponding author: C.A. Fuzo

E-mail: cafuzo@usp.br

Genet. Mol. Res. 7 (4): 986-999 (2008)

Received May 29, 2008

Accepted June 25, 2008

Published October 7, 2008

\begin{abstract}
The antimicrobial peptide indolicidin (IND) and the mutant CP10A in hydrated micelles were studied using molecular dynamics simulations in order to observe whether the molecular dynamics and experimental data could be sufficiently correlated and a detailed description of the interaction of the antimicrobial peptides with a model of the membrane provided by a hydrated micelle system could be obtained. In agreement with the experiments, the simulations showed that the peptides are located near the surface of the micelles. Peptide insertions agree with available experimental data, showing deeper insertion of the mutant compared with the peptide IND. Major insertion into the hydrophobic core of the micelle by all tryptophan and mutated residues of CP10A in relation to IND was observed. The charged residues of the terminus regions of both peptides present similar behavior, indicating that the major differences in the interactions with the micelles of the peptides IND and CP10A occur in the case of the hydrophobic residues.
\end{abstract}

Key words: Antimicrobial peptides; Indolicidin; Indolicidin mutant; Dodecylphosphocholine micelle; Peptide-micelle interaction;

Molecular dynamics simulation 


\section{INTRODUCTION}

Antimicrobial peptides (AMPs) are important elements of the innate immune defense system of many organisms (Hancock and Diamond, 2000; Dutton et al., 2002; Gallo, 2005). AMPs are generally defined as peptides formed by less than 50 amino acid residues, with an overall positive charge, imparted by the presence of multiple lysine and arginine residues and by a substantial portion of hydrophobic residues (Powers and Hancock, 2003). It is well known that the main mechanism for the rapid killing of microbial pathogens by a large number of AMPs is due to the perturbations that AMPs induce in the microbial cell membrane (Gordon et al., 2005). Since AMPs have no specific target, the development of novel therapeutic agents based on these kinds of peptides may be a way to overcome microorganism resistance to conventional antibiotics (Zasloff, 2002; McPhee and Hancock, 2005).

Indolicidin (IND) has caught researchers' attention because it is the shortest natural AMP and it is also a very efficient antimicrobial agent (Selsted et al., 1992). IND has been isolated from bovine neutrophils by Selsted et al. (1992). It consists of 13 amino acid residues (ILPWKWPWWPWRR-NH ${ }_{2}$ ), it is amidated at its C-terminus, and it contains a large proportion of tryptophan residues (39\%) (Selsted et al., 1992). Like many other AMPs, it displays activity against a broad range of microorganisms such as Gram-positive and Gram-negative bacteria, fungi, and protozoa (Chan et al., 2006). However, IND is toxic to lymphocytes and erythrocytes (Falla and Hancock, 1997). IND has been the target of many studies because its small size facilitates its synthesis, not to mention the fact that tryptophan residues are used as key residues in experimental measurements (Mateo et al., 2006). Therefore, several efforts have been made toward decreasing the toxicity and increasing the cell selectivity of this peptide (Sitaram and Nagaraj, 1999; Chan et al., 2006). Modified versions of IND, including a Pro to Ala mutant (ILAWKWAWWAWRR-NH ${ }_{2}$ ) called CP10A, have been the object of many studies (Subbalakshmi et al., 1996; Friedrich et al., 2001; Halevy et al., 2003). Interestingly, the lytic activity of the CP10A mutant is higher on human erythrocytes compared with that of IND (Halevy et al., 2003). Experimental studies on IND and CP10A in mimetic membrane systems have shown that these peptides interact with membranes, resulting in deeper insertion of the mutated peptide CP10A compared with the natural one (Subbalakshmi et al., 1998; Zhang et al., 2001; Halevy et al., 2003).

The structures of the peptides IND (Rozek et al., 2000) and CP10A (Friedrich et al., 2001) have been determined by NMR experiments in the presence of dodecylphosphocholine (DPC) micelles. CP10A adopts a short helical structure instead of the extended structure of the IND peptide (Friedrich et al., 2001). Details of the peptide-micelle interactions have not been elucidated to date, despite all the knowledge about the structure and insertion of these two peptides into membrane models gained from these experiments. The different interactions these peptides show with the micelles should help establish structure activity relationships. Although, details of peptide insertion and positioning in the membranes are of particular interest so that more realistic models concerning the action of AMPs can be achieved, knowledge about models of interactions between AMPs and membranes is still subject to the limited information obtained from experiments. In this sense, computational techniques have been used to solve many problems concerning biological systems and to complement experimental observations (Murakami et al., 2005; Brancaleoni et al., 2006; Mazzé et al., 2007; Fuzo et al., 2008). Among the various computational techniques, molecular dynamics simulation (Alder and Wainwright, 1957) is a powerful tool that provides detailed configurational 
and dynamic information at the atomic level.

Here we present the results of molecular dynamics studies on the antimicrobial peptides IND and CP10A and their interaction with hydrated DPC micelles. Micelles were used instead of bilayers so that comparisons with experimental data could be made. Another fact that must be taken into account is that the dynamics of micelles is faster than the dynamics of membranes, so simulation convergence is reached with lower computational cost (Khandelia et al., 2006). The aim of the present study was to observe whether the molecular dynamics and experimental data are sufficiently correlated, so that a detailed description of the interaction of the antimicrobial peptide IND and CP10A with a model of the zwitterionic interface provided by a hydrated micelle system could be obtained.

\section{SIMULATION DETAILS}

Simulations were carried out with the GROMACS 3.3.1 simulation package (van der Spoel et al., 2005a) in a Linux cluster. The ffgmx force field (van der Spoel et al., 2005b) was used for the peptides and the model SPC for the water molecules (Berendsen et al., 1981), while the force field parameters for the DPC molecule (Tieleman et al., 2000) were taken from http://moose. bio.ucalgary.ca/. Peptides and detergent covalent bonds were constrained by the LINCS algorithm (Hess et al., 1997). The SETTLE algorithm (Miyamoto and Kollman, 1992) was employed to maintain the structural stability of the water molecules. The temperature $(300 \mathrm{~K})$ and pressure $(1$ atm) of the systems were regulated by the Berendsen's algorithms (Berendsen et al., 1984), with time constants of 0.1 and $1 \mathrm{ps}$, respectively. Short range and electrostatic interactions were cut-off at a distance of $1.0 \mathrm{~nm}$. Long range electrostatic interactions were computed using the particle mesh Ewald summation method (Darden et al., 1993).

The structures of the peptides IND (Rozek et al., 2000) and CP10A (Friedrich et al., 2001) deposited in the Protein Data Bank (http://www.rcsb.org/pdb) with the pdb codes 1G89 and 1HR1, respectively, were used as the initial structures of the peptides in the construction of the peptide/micelle/water systems. These experimental peptide structures were determined in the presence of DPC micelles. The structure of a micelle, constituted by 65 DPC molecules, hydrated by 6305 water molecules obtained after a molecular dynamics simulation of about $1 \mathrm{~ns}$ in a cubic simulation box (Tieleman et al., 2000), was used as the initial estimate of the micelle/water system. The cubic box of the hydrated DPC micelle was enlarged from 6.08 to about $7.00 \mathrm{~nm}$, to increase the distances between the micelle and its images. The micelle center of mass (CM) was located in the center of the simulation cubic box, and water molecules were added to fill the extra region of the box so that the final number of water molecules was 10,219. This system was first energy-minimized using a steepest descent algorithm and then simulated for $10 \mathrm{~ns}$ with integration steps of $2 \mathrm{fs}$. The systems formed by the peptides inserted into the micelles in aqueous medium were constructed by locating the $\mathrm{CM}$ of the peptide in the same position as the $\mathrm{CM}$ of the micelle. The water molecules and the DPC molecules in bad contacts with the peptide atoms were removed. Negative $\mathrm{Cl}^{-}$ions were inserted into electrostatically favorable positions, in order to preserve the local electroneutrality. The constitutions of each system are listed in Table 1. The systems were firstly energy-minimized by the same steepest descent algorithm mentioned above, and $0.5 \mathrm{~ns}$ of molecular dynamics were then conducted with restrictions on the peptide atomic coordinates using the energy profiles as control criteria. Finally, the simulations were carried out without restrictions for $120 \mathrm{~ns}$ with a time step of $2 \mathrm{fs}$. The coordinates of all the atoms were re- 
corded every 5 ps for further analysis, which was carried out using GROMACS tools and specific programs developed in our laboratory.

Table 1. The primary structure of the peptides, the peptide liquid charge, the number of dodecylphosphocholine (DPC) molecules and the number of water molecules in each system.

\begin{tabular}{lcccc}
\hline Peptide & Primary sequence & Charge & $\begin{array}{c}\text { Number of DPC } \\
\text { molecules }\end{array}$ & $\begin{array}{c}\text { Number of water } \\
\text { molecules }\end{array}$ \\
\hline IND & ILPWKWPWWPWRR-NH & +4 & 60 & 10187 \\
CP10A & ILAWKWAWWAWRR-NH & +4 & 61 & 10210 \\
\hline
\end{tabular}

$\mathrm{IND}=$ indolicidin.

\section{RESULTS AND DISCUSSION}

The behavior of the micelles was quantified using properties such as water insertion into the hydrophobic core of the micelle and micelle shape. The presence of water molecules in the micelles was measured by water density, $\rho_{\mathrm{w}}\left(\mathrm{r}_{\mathrm{M}}\right.$ ), where $\mathrm{r}_{\mathrm{M}}$ is the distance from the micelle $\mathrm{CM}$ (Figure 1). The $\rho_{\mathrm{w}}\left(\mathrm{r}_{\mathrm{M}}\right)$ value indicates that the water molecules are unable to penetrate the hydrophobic core of the micelles and that the core radius is about $1.2 \mathrm{~nm}$ in all cases. The $\rho_{\mathrm{w}}\left(\mathrm{r}_{\mathrm{M}}\right)$ value increases from $\mathrm{r}_{\mathrm{M}}=1.2 \mathrm{~nm}$ to about $2.6 \mathrm{~nm}$ where bulk water density is achieved. These results agree with the densities (Wymore et al., 1999; Tieleman et al., 2000) obtained in simulations of pure hydrated DPC micelles. The differences between the $\rho_{\mathrm{w}}\left(\mathrm{r}_{\mathrm{M}}\right)$ values for the systems containing the IND and CP10A peptides and the $\rho_{\mathrm{w}}\left(\mathrm{r}_{\mathrm{M}}\right)$ values of the pure micelle system are not significant because they are due to 1.5 water molecules, on average, along the interface region (from 1.2 at $2.6 \mathrm{~nm}$ ), while they are close to zero in the other regions. Consequently, the main structural characteristics of the micelles, namely the hydrophobic core close to the micelle center and the structure of the interfacial regions, are maintained in the presence of the peptides. The sphericity of the micelles was checked using the ratio between three main inertia moments. These ratios are equal to one for a sphere and 1.2:1.1:1 in the cases of IND and CP10A. These ratios point to an asymmetrical structure for the

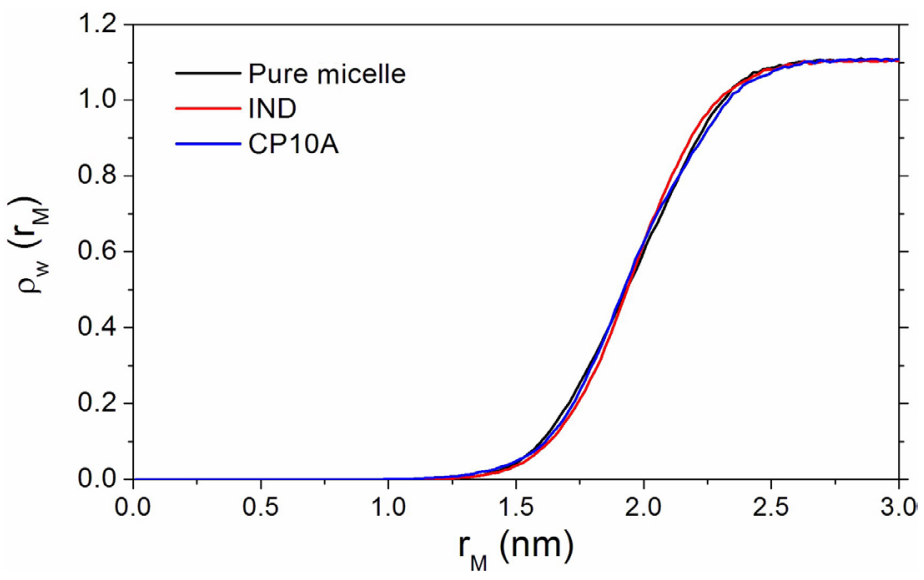

Figure 1. $\rho_{\mathrm{w}}\left(\mathrm{r}_{\mathrm{M}}\right)$ of the water molecules relative to the center of mass of the micelles. IND = indolicidin. 
micelles when they are different from one. Therefore, the presence of the IND and CP10A peptides does not result in micelle disruption since the ratios are equal to the ones found for micelle/water systems and in independent simulations of pure hydrated DPC micelles carried out by Wymore et al. (1999) and Tieleman et al. (2000).

The initial and final structures of the peptides in the micelles are shown in Figure 2. Comparing their initial and final configurations, it is clear that changes in peptide location and residue orientations occur. The peptide motions are directed from the initial position of the peptide/micelle system center to the interface as seen from the distances between the CM of the peptides and the $\mathrm{CM}$ of the micelles $\left(\mathrm{d}_{\text {pep-mic }}\right)$ shown in Figure 3. Initially, the $\mathrm{CM}$ of the peptides was coincident with the $\mathrm{CM}$ of the micelles. After the energy minimization steps the positions of both CMs were not coincident any more, accounting for the $\mathrm{d}_{\text {pen-mic }}$ values different from zero at time zero in Figure 3. Diffusion takes place more rapidly for the IND peptide since its CM is $1 \mathrm{~nm}$ away from the CM of the micelle after $20 \mathrm{~ns}$, while the same distance is observed only after $70 \mathrm{~ns}$ for CP10A. For times longer than $20 \mathrm{~ns}$, some fluctuations are observed in the $\mathrm{d}_{\text {pep-mic }}$ of IND in the interval 0.8 to $1.2 \mathrm{~nm}$. These fluctuations are due to peptide accommodation in the micelle. $d_{\text {pep-mic }}$ fluctuations decrease and the distance stabilizes at about $1 \mathrm{~nm}$ during the last $20 \mathrm{~ns}$ of the simulation. Variations in the

IND
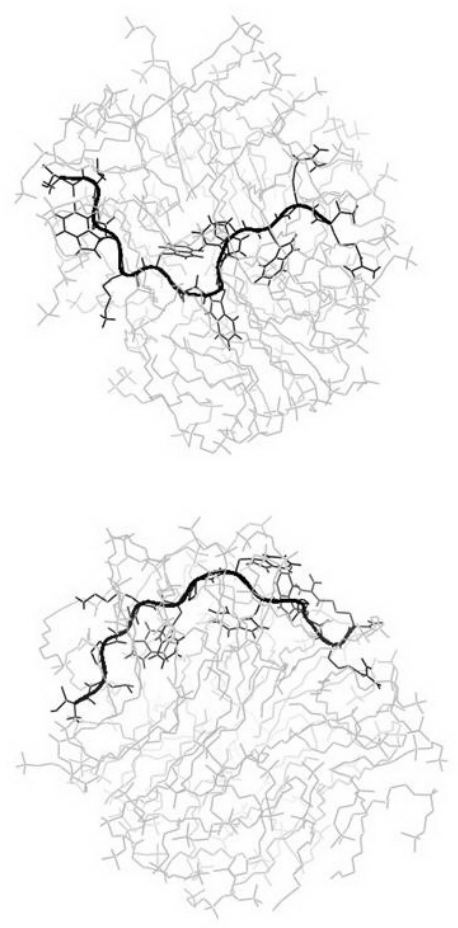

CP10A
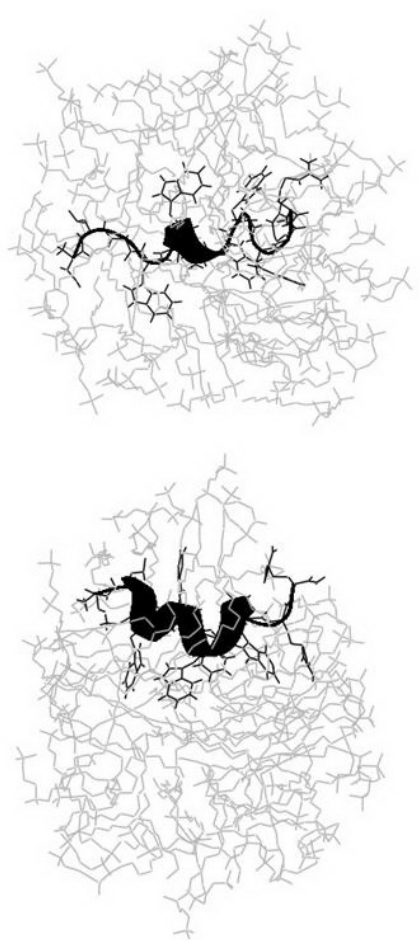

Figure 2. The starting (top) and the final (bottom) conformations of the peptides and micelles. Water molecules have been removed for better visualization. The final snapshots were taken after $120 \mathrm{~ns}$. IND = indolicidin. 


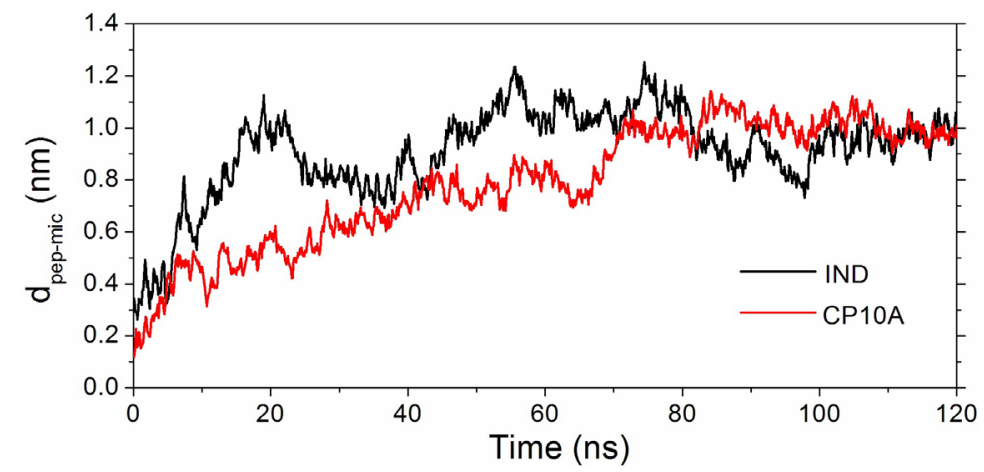

Figure 3. Distance between the center of mass of the peptides and the center of mass of the dodecylphosphocholine micelles during the simulations.

$\mathrm{d}_{\text {pep-mic }}$ profiles are smaller for the CP10A peptide than for the IND peptide. The CM of the CP10A peptide migrates slowly to the micelle surface, within $70 \mathrm{~ns}$, stabilizing at about $1 \mathrm{~nm}$ until the end of the simulation. The more restricted CP10A helical structure does not allow for the same easy accommodation observed in the case of the IND peptide.

The mean distances between the CM of each residue and the CM of the micelles, $d_{\text {res-mic }}$, plotted in Figure 4, are larger than $1.2 \mathrm{~nm}$ for many IND residues. This is exactly the smallest distance that the water molecules can reach, Figure 1 . The $\mathrm{d}_{\text {res-mic }}$ of the residue Lys 5 is $1.8 \mathrm{~nm}$; i.e., the charged groups of this residue tend to approximate the water phase, where the attractive energies are more favorable than in the hydrophobic region of the micelle. The overall IND structure is an extended chain that maintains its skeleton at about the same distance from the $\mathrm{CM}$ of the micelle, with the charged and polar lateral groups directed toward the micelle surface and many of the nonpolar lateral groups directed toward the hydrophobic core of the micelle. The CP10A helical conformation explains the oscillations in $\mathrm{d}_{\text {res-mic }}$ values seen in Figure 4 , while the charged groups are located in the micelle as observed as the case of IND, see Figure 2. The helical conformation seems to promote better insertion of the nonpolar residues since Trp4 and Trp 8 are distant only 1.02 and $0.55 \mathrm{~nm}$ from the center of the micelle, respectively (see Figure 4). Insertion of Trp4 and the CP10A helical conformation place the Lys5 residue closer to the micelle center compared with the IND Lys5 residue. These results show that the whole extension of the IND peptide migrates to

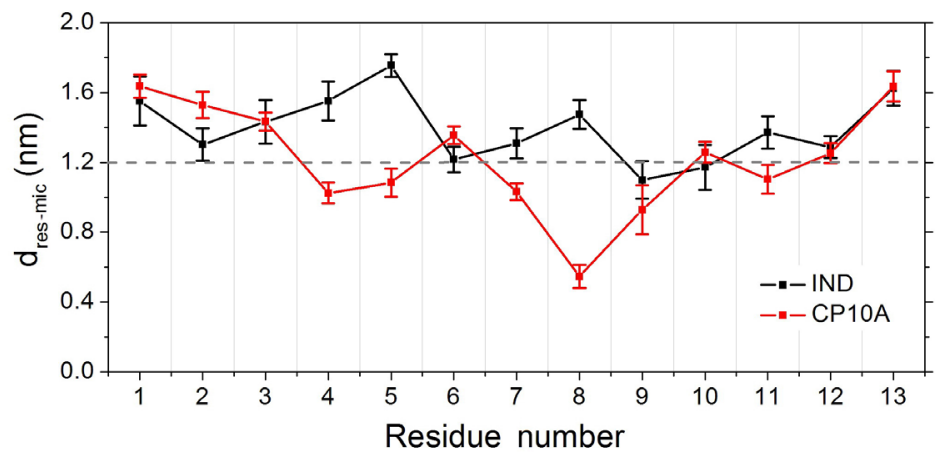

Figure 4. $d_{\text {res-mic }}$ of the indolicidin (IND) and CP10A peptides calculated from 115 to $120 \mathrm{~ns}$. 
the micelle/water interface, while the CP10A peptide remains more inserted into the micelle as a consequence of its helical conformation. These results agree with the membrane location probed by fluorescence spectroscopy using spin-labeled lipids, which indicates that the interfacial membrane/ water location is preferred for IND (Rozek et al., 2000) and CP10A (Friedrich et al., 2001).

The $\mathrm{C}_{\alpha}$ root-mean-square deviations (RMSD) of the two peptides in relation to the initial structures and the fits of the initial and final conformations of the peptides are shown in Figure $5 \mathrm{~A}$ and $\mathrm{B}$, respectively. The RMSD of both peptides initially increase to values close to $0.2 \mathrm{~nm}$. The IND RMSD value presents an oscillating behavior $(0.05 \mathrm{~nm})$ until $50 \mathrm{~ns}$, before it begins a greater oscillation $(0.2 \mathrm{~nm})$ until $110 \mathrm{~ns}$. This indicates that the peptide encounters some difficulty in escaping from a local energy minimum before it reaches more stable conformations. This observation is corroborated by the former results on the distances to the micelle center, which showed that the peptide moves to the interfacial region and simultaneously changes its structure. From 110 ns until the end of the simulation, the RMSD value remains stable and oscillates at around $0.24 \mathrm{~nm}$. The RMSD value for CP10A is very stable from the beginning of the simulation process until $120 \mathrm{~ns}$, since it oscillates around $0.18 \mathrm{~nm}$ during this interval. The fast stabilization of the CP10A structure in the micelle is a direct consequence of the fact that its helix allows its various residues to find energetically appropriate locations according to their polarity properties, as already discussed in the case of the $d_{\text {res-mic }}$ results. The NMR experiments showed that the IND peptide has an extended structure (Rozek et al., 2000) and that the structure of the CP10A peptide is a helical one (Friedrich et al., 2001) in the presence of the DPC molecules. The IND peptide is more flexible due to its extended structure, resulting in major RMSD fluctuations, even at times when the ordered helical CP10A peptide structure remains stable, as seen from their RMSD values. The low RMSD values and the fits of the initial and final configurations of both peptides (Figure 5) show that the structures obtained from the simulations are not essentially different from the structures obtained experimentally (Rozek et al., 2000; Friedrich et al., 2001).

A

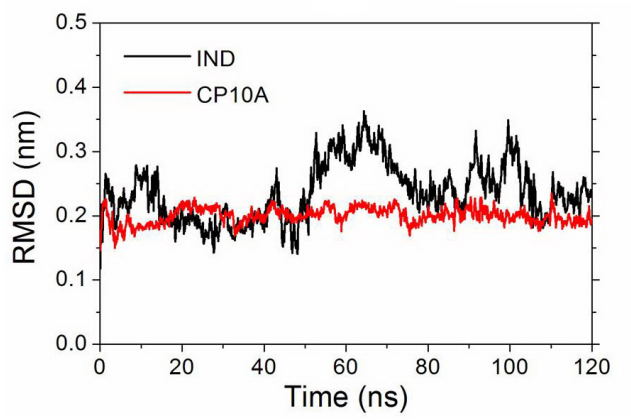

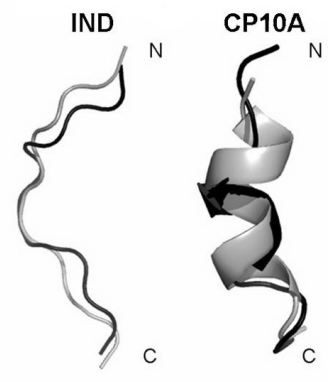

Figure 5. A. Time profile of the backbone root-mean-square deviations (RMSD) in relation to the initial structures. B. Initial (in black) and final (in gray, $\mathrm{t}=120 \mathrm{~ns}$ ) fitted structures of the two peptides. IND $=$ indolicidin.

The intramolecular hydrogen bonds (HBs) were identified using criteria such as angle between the donor-hydrogen and donor-acceptor vectors no larger than $30^{\circ}$ and a maximum donor-acceptor distance shorter than $0.35 \mathrm{~nm}$. No intramolecular HB was found in the IND peptide. This is because its extended structure does not allow the approximation of the donor- 
acceptor groups that is necessary for HB formation. On the other hand, the helical structure of the CP10A peptide is conserved by a typical HB network formed only through the main chain atoms, as listed in Table 2. The HBs found for CP10A are typical of the $\alpha(n \rightarrow n+4)$ and $3_{10}$ $(n \rightarrow n+3)$ helices (Branden and Tooze, 1999). The $n \rightarrow n+4$ HBs frequently appear all over the extension of the peptide, with occurrences going from 29 to $92 \%$, while occurrences of the three $n \rightarrow n+3$ HB are lower than 48\%. The stability of the structure of the CP10A peptide is thus explained by these main chain HBs, which stabilize the $\alpha$-helix conformation according to both experimental and simulation results.

\begin{tabular}{|c|c|c|c|}
\hline Donor & Acceptor & Occurrence (\%) & Type \\
\hline Trp-6(NH) & Leu-2(CO) & 91.7 & $\mathrm{n} \rightarrow \mathrm{n}+4$ \\
\hline Ala-10(NH) & Trp-6(CO) & 85.0 & $\mathrm{n} \rightarrow \mathrm{n}+4$ \\
\hline Ala-7(NH) & Ala-3(CO) & 84.0 & $\mathrm{n} \rightarrow \mathrm{n}+4$ \\
\hline Arg-13(NH) & Trp-9(CO) & 55.5 & $\mathrm{n} \rightarrow \mathrm{n}+4$ \\
\hline Trp-9(NH) & Trp-6(CO) & 47.6 & $\mathrm{n} \rightarrow \mathrm{n}+3$ \\
\hline Trp-9(NH) & Ala-7(CO) & 46.6 & $\mathrm{n} \rightarrow \mathrm{n}+4$ \\
\hline Trp-8(NH) & Trp-4(CO) & 29.3 & $\mathrm{n} \rightarrow \mathrm{n}+4$ \\
\hline Arg-12(NH) & Trp-9(CO) & 26.8 & $\mathrm{n} \rightarrow \mathrm{n}+3$ \\
\hline Arg-13(NH) & Ala-10(CO) & 22.3 & $\mathrm{n} \rightarrow \mathrm{n}+3$ \\
\hline Trp-8(NH) & Lys-5(CO) & 18.8 & $\mathrm{n} \rightarrow \mathrm{n}+3$ \\
\hline
\end{tabular}

Another important intramolecular interaction that contributes to stabilization of the tertiary structure is the cation- $\pi$ interaction (Gallivan and Dougherty, 1999) that may take place between the positive guanidinium ion of the Arg residues and the side chain of the Trp residues, where the electronic density due to the $\pi$ bonds is seen as a liquid negative charge at short distance. The cation- $\pi$ interactions were identified from the distance between the $\mathrm{CM}$ of the Arg guanidinium ion and the Trp side chain and by the angle between their planes during the last $5 \mathrm{~ns}$ of the simulations. The distances and angles of cation- $\pi$ interactions must be below $0.5 \mathrm{~nm}$ and between $0-45^{\circ}$, respectively (Aliste et al., 2003; Khandelia and Kaznessis, 2007). A cation- $\pi$ interaction between Trp11 and Arg 13 is observed in the IND peptide, with mean distance and angle of $0.43 \pm 0.14 \mathrm{~nm}$ and $30.7 \pm 21.0^{\circ}$, respectively, and a mean energy of $-39 \pm 17 \mathrm{~kJ} \mathrm{~mol}^{-1}$. Khandelia and Kaznessis (2007) observed the same cation- $\pi$ interaction with energy equal to $-66 \pm 16 \mathrm{~kJ} \mathrm{~mol}^{-1}$ for the IND peptide in DPC micelles, despite the use of a different force field and a smaller system. This cation- $\pi$ interaction is a stabilizing factor in the structure of IND. A cation- $\pi$ interaction between the residues Trp11 and Arg 12 is observed in the CP10A peptide, with a mean distance of $0.48 \pm 0.04 \mathrm{~nm}$, a mean angle equal to $31.1 \pm 11.8^{\circ}$ and a mean energy of $-24 \pm 6 \mathrm{~kJ} \mathrm{~mol}^{-1}$. These values are consistent with literature data (Khandelia and Kaznessis, 2007). The cation- $\pi$ interaction is more attractive in the IND peptide than in $\mathrm{CP} 10 \mathrm{~A}$ as a consequence of the presence of the $\alpha$-helix, which makes the approximation of the Arg guanidinium ion and of the Trp side chain in CP10A difficult.

The UV spectrum of Trp residues is very sensitive to the environment so emission/ absorption spectra of the Trp residues were used in order to study the peptide-lipid interaction in the cases of IND and CP10A (Subbalakshmi et al., 1998; Rozek et al., 2000; Friedrich et 
al., 2001; Zhang et al., 2001; Halevy et al., 2003). The $d_{\text {res-mic }}$ values demonstrate that some Trp residues are closer to the micelle center than others in both the IND and CP10A peptides. However, these results do not reveal how many atoms of the hydrophobic core of the micelles surround the Trp residues. Such information is given by the radial distribution functions, $g(r)$, for the pairs formed between the $\mathrm{C}$ or $\mathrm{N}$ side chain atoms of the Trp residues and the carbon atoms of the hydrophobic chains of the DPC molecules. These profiles are plotted in Figure 6. The well-defined first peak in the IND g(r) of Trp4, Trp6, Trp9, and Trp11 at $0.48 \mathrm{~nm}$ indicates that the side chains of these residues are surrounded by a fairly good structure constituted by atoms of the hydrophobic region of the micelles in this region. The same conclusion is also valid for Trp8 of the CP10A peptide. Some Trp residues of the IND peptide have a more defined $g(r)$ than others since a minor intensity in the first peak is observed, for example, for Trp8. The intensities of the $\mathrm{g}(\mathrm{r})$ peaks of the CP10A peptide show that the neighborhood, formed by the hydrophobic region of the micelle, of the Trp residues is fairly structured. The aspects of the $\mathrm{g}(\mathrm{r})$ profiles reveal that the medium is generally fairly structured around the Trp residues. These results are corroborated by the fluorescence, spin-labeled lipid probe and Langmuir monolayer experiments carried out with these two peptides when they are inserted in modeled membranes (Subbalakshmi et al., 1998; Zhang et al., 2001; Halevy et al., 2003). In particular, the simulation results are consistent with the experimental ones, showing that the $\mathrm{CP} 10 \mathrm{~A}$ peptide is inserted deeper into the membranes compared with the IND peptide.
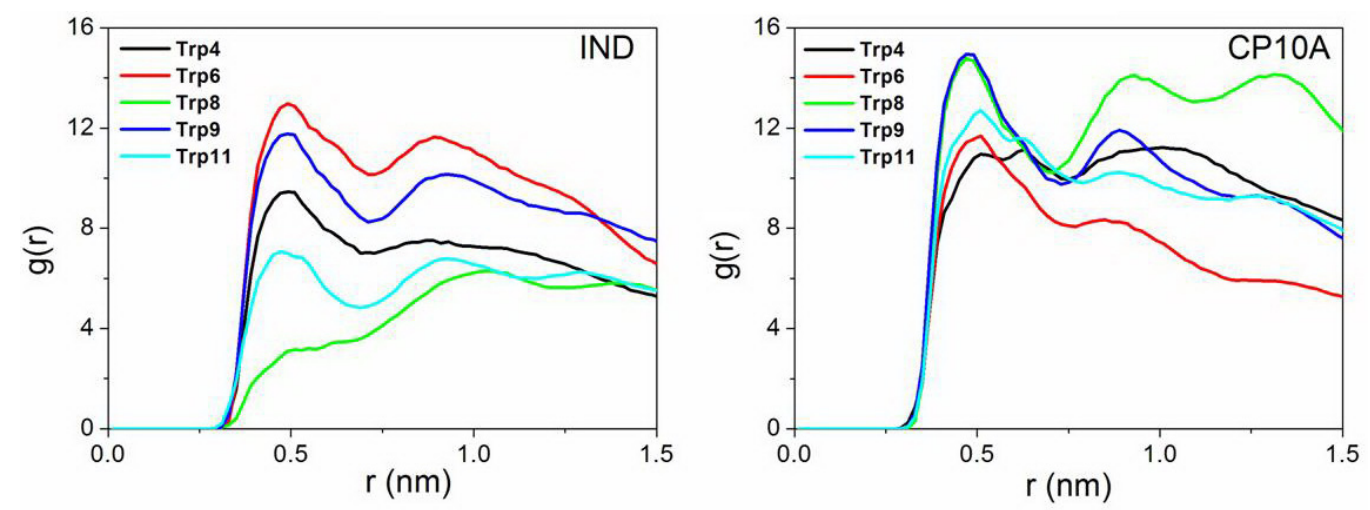

Figure 6. $\mathrm{g}(\mathrm{r})$ for the pairs formed by the $\mathrm{C}$ and $\mathrm{N}$ side chain atoms of the Trp residues of indolicidin (IND) and CP10A and the carbon atoms of the hydrophobic chains of the dodecylphosphocholine molecules.

The $g(r)$ of the other hydrophobic residues (Ile, Leu, Pro, and Ala) were calculated between the carbon atoms of the side chains and the carbon atoms of the hydrophobic chains of the DPC molecules in the same way as was done for the Trp residues (Figure 7). The first g(r) peaks for the IND Leu2, Pro3, Pro7, and Pro10 residues are observed at the same position $(0.48 \mathrm{~nm})$ found for the Trp residues, while the residue Ile1 has no defined peak in its $\mathrm{g}(\mathrm{r})$ function. These results are consistent with the Ile $1 \mathrm{~d}_{\text {res-mic }}$ value of about $1.55 \mathrm{~nm}$, since some water molecules are found at this distance (Figure 1). Well-defined $\mathrm{g}(\mathrm{r})$ first peaks are observed for $\mathrm{CP} 10 \mathrm{~A}$ residues at the same $0.48 \mathrm{~nm}$ distance, except for the Leu2 residue. The intensities of 

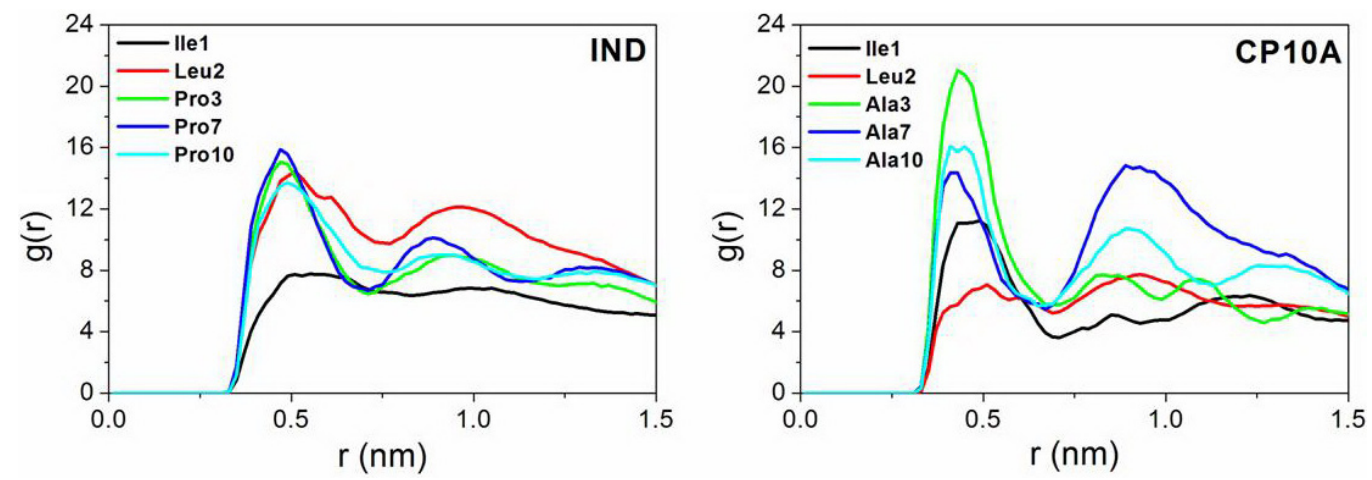

Figure 7. $\mathrm{g}(\mathrm{r})$ for the pairs formed by the $\mathrm{C}$ side chain atoms of the Ile, Leu, Pro, and Ala residues of indolicidin (IND) and CP10A and the carbon atoms of the dodecylphosphocholine hydrophobic chains.

the first and second peaks of residues 3, 7 and 10 at the mutation points (Pro $\rightarrow$ Ala) (Figure 7) reveal that the first $\mathrm{g}(\mathrm{r})$ peak is more defined for residues 3 and 10 in the CP10A peptide and for residue 7 in IND. Although there are no significant differences in the intensities of the first $\mathrm{g}(\mathrm{r})$ peaks of residues 7 and 10, the second peaks of these residues are more defined and intense in CP10A than in IND. This indicates that the insertion of residues 3, 7 and 10 into the hydrophobic region of the micelle is better in the CP10A peptide than in the IND peptide. It had already been underscored that experimental data (Subbalakshmi et al., 1998; Zhang et al., 2001; Halevy et al., 2003) indicate that the Trp residues in CP10A are inserted deeper into the hydrophobic regions of the modeled membrane. However, the $g(r)$ profiles allow one to conclude that the Ala residues at the mutation points also interact more effectively with the hydrophobic region of the micelle than the corresponding Pro residues in the IND peptide.

The cationic side chains, including the N-terminal group, interact strongly with the negatively charged phosphate groups of the DPC molecules and also with the water molecules. These interactions generate the ordering of the phosphate groups and water molecules around the positively charged groups, as detected by means of various $\mathrm{g}(\mathrm{r})$ profiles. The radial distribution functions for the pair phosphorus atom/nitrogen atoms of the N-terminus and of the Lys5 amine groups are plotted in Figure 8A. The g(r) for the carbon atoms of the Arg12 and Arg13 guanidinium groups with the phosphorus atoms of the DPC molecules are depicted in Figure 8B. In both figures, the first defined peaks are observed at $0.37 \mathrm{~nm}$ for all amine groups and at 0.43 $\mathrm{nm}$ for the guanidinium groups. The water neighborhood of the same atoms (nitrogen for amine groups and carbon for guanidinium groups) is described through the corresponding $\mathrm{g}(\mathrm{r})$ plotted in Figure 9A for the amine groups and in Figure 9B for the guanidinium groups. The first hydration shell results in a maximum at 0.29 and $0.36 \mathrm{~nm}$ for the amine and the guanidinium groups, respectively. The numbers of the first neighbors of phosphorus atoms $\left(\mathrm{N}_{\mathrm{p}}\right)$ and water molecules $\left(\mathrm{N}_{\mathrm{w}}\right)$ calculated from the $\mathrm{g}(\mathrm{r})$ of Figures 8 and 9 are listed in the Table 3 . The mean composition of the first shells around the amine groups of the N-terminus of both peptides and Lys 5 of CP10A is about two phosphate groups and one water molecule. Three water molecules and about one phosphate group are found in the solvation shell of the Lys5 amine group of IND. This number of water molecules is consistent with the high $\mathrm{d}_{\text {res-mic }}$ value (Figure 4), which showed that this resi- 
A

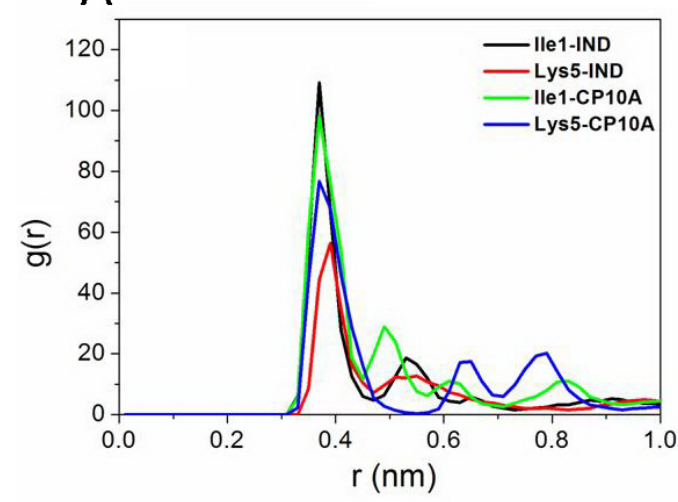

B

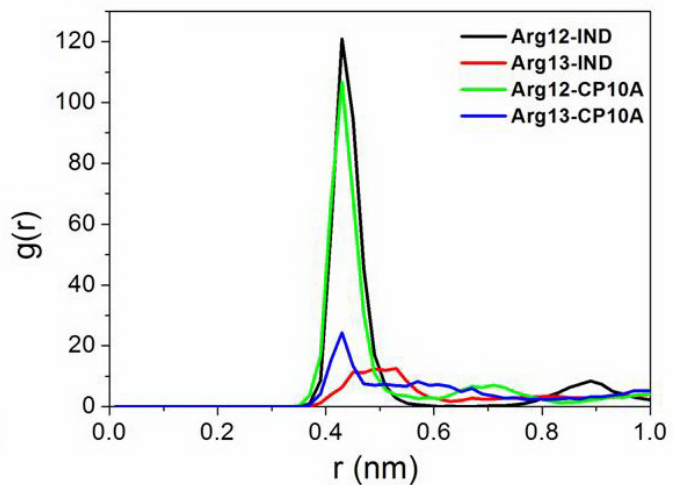

Figure 8. A. $g(r)$ for the pair nitrogen atoms of the N-terminus and of the Lys5 amine groups and the dodecylphosphocholine phosphorus atoms. B. $g(r)$ for the pair phosphorus atom/carbon atoms of the Arg 12 and $\operatorname{Arg} 13$ guanidinium groups. IND = indolicidin.
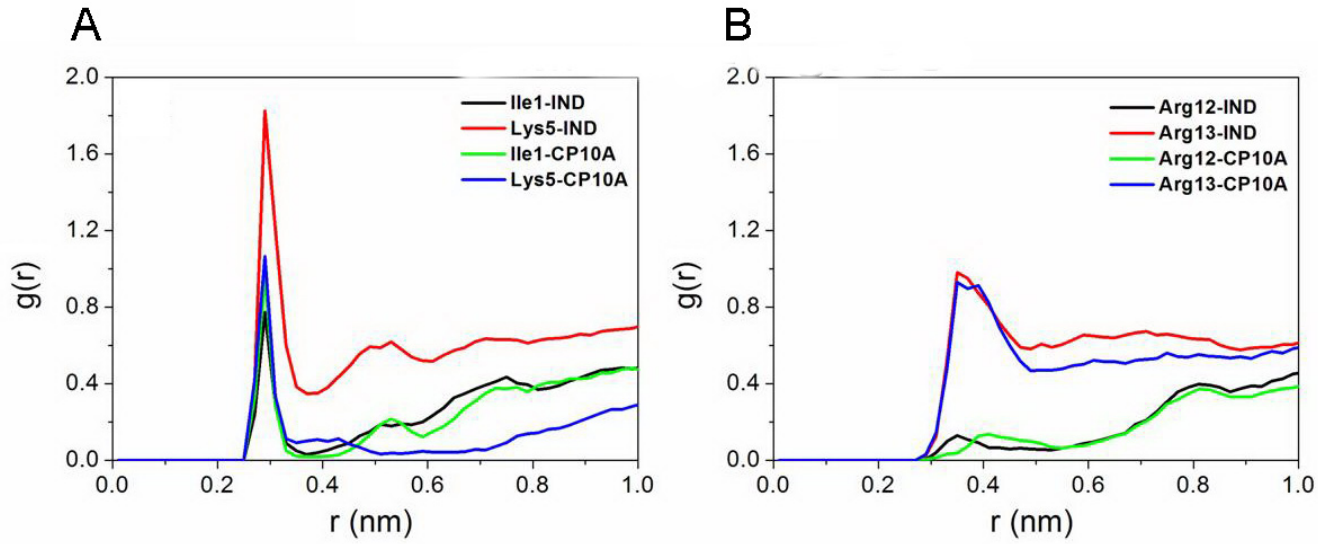

Figure 9. A. $g(r)$ for the pair oxygen atoms of the water molecules and the nitrogen atoms of the N-terminus and of the Lys5 amine groups. B. $g(r)$ for the pair oxygen atoms of the water molecules and the carbon atoms of the Arg 12 and $\operatorname{Arg} 13$ guanidinium groups. IND = indolicidin.

Table 3. Numbers of neighbors of the positively charged groups of the indolicidin (IND) and CP10A peptides.

\begin{tabular}{lcccc}
\hline & \multicolumn{2}{c}{ IND } & \multicolumn{2}{c}{ CP10A } \\
\cline { 2 - 5 } & $\mathrm{N}_{\mathrm{P}}$ & $\mathrm{N}_{\mathrm{W}}$ & $\mathrm{N}_{\mathrm{P}}$ & $\mathrm{N}_{\mathrm{W}}$ \\
\hline N-terminus & 1.73 & 0.93 & 2.10 & 1.02 \\
Lys5 & 1.13 & 3.02 & 1.88 & 1.29 \\
Arg12 & 3.00 & 0.81 & 2.77 & 0.97 \\
Arg13 & 0.92 & 7.25 & 1.10 & 6.94 \\
\hline
\end{tabular}


due is located close to the interface between the micelle and the water phase. The well-defined $\mathrm{g}(\mathrm{r})$ peaks and the 3 phosphorus groups around the guanidinium groups of the Arg 12 residues indicate that these residues are largely surrounded by the head groups of the DPC molecules; i.e., they are inserted into the zwitterionic head of these amphiphilic molecules. On the other hand, the guanidinium groups of the Arg13 residues are inserted mainly into the water phase. These remarks are consistent with the $d_{\text {res-mic }}$ values of about 1.2 and $1.65 \mathrm{~nm}$ found for the $\operatorname{Arg} 12$ and Arg13 residues, respectively. All these results show that, in both peptides, the neighborhoods of the positively charged groups of the N-terminus, $\operatorname{Arg} 12$ and Arg13, are very similar. The corollary is that the major differences in the interaction between the peptides IND and CP10A and the micelles must lie mainly on the hydrophobic residues, as described earlier.

\section{CONCLUSION}

The results of the molecular dynamics simulations of the interaction between the IND and the mutant CP10A antimicrobial peptides with DPC micelles were presented and were found to be consistent with experimental data. To the best of our knowledge, these simulations (120 ns) are longer than the simulations of peptides into micelles reported in the literature. The peptide insertions observed here are favorably compared with the available experimental data, and corroborate a deeper insertion into the micelle in the case of the mutant CP10A compared with the peptide IND. However, the extended structure of the IND peptide and the helical structure of the CP10A peptide are maintained, as had been found experimentally. The corroboration between experimental and simulation data suggests that the simulations are able to describe the interaction between the IND and CP10A antimicrobial peptides with DPC micelles correctly, and the data that are not available from experience are reliable. In this context, we observed major insertion of the CP10A tryptophan residues into the hydrophobic core of the micelle compared with the IND tryptophan residues. The residues in the mutation points are better inserted into this region of the micelle than the IND residues. Finally, the charged residues of the terminus regions in both peptides present similar behavior, indicating that the major differences in interaction of the IND and CP10A peptides with the micelles occur in the case of hydrophobic residues. The higher hemolytic activity of the CP10A peptide compared with the wild peptide is explained by the fact that $\mathrm{CP} 10 \mathrm{~A}$ must be able to penetrate the cellular membrane deeper than IND, thus resulting in a more powerful destruction of the local structures that preserve the integrity of this kind of cellular membranes.

\section{ACKNOWLEDGMENTS}

Research supported by Fundação de Amparo à Pesquisa do Estado de São Paulo (FAPESP), Conselho Nacional de Desenvolvimento Científico e Tecnológico (CNPq) and Coordenação de Aperfeiçoamento de Pessoal de Nível Superior (CAPES).

\section{REFERENCES}

Alder BJ and Wainwright TE (1957). Phase transition for a hard sphere system. J. Chem. Phys. 27: 1208-1209.

Aliste MP, MacCallum JL and Tieleman DP (2003). Molecular dynamics simulations of pentapeptides at interfaces: salt 
bridge and cation- $\pi$ interactions. Biochemistry 42: 8976-8987.

Berendsen HJC, Postma JPM, van Gunsteren WF and Hermans J (1981). Interaction models of water in relation to protein hydration. In: Intermolecular Forces (Pullman B and Reidel D, eds.). Reidel Publishing Company, Dordrecht, 331-342.

Berendsen HJC, Postma JPM, van Gusteren WF, Dinola A, et al. (1984). Molecular dynamics with coupling to an external bath. J. Chem. Phys. 81: 3684-3690.

Brancaleoni GH, Lourenzoni MR and Degreve L (2006). Study of the influence of ethanol on basic fibroblast growth factor structure. Genet. Mol. Res. 5: 350-372.

Branden C and Tooze J (1999). Introduction to Protein Structure. Garland Publishing Inc., New York.

Chan DI, Prenner EJ and Vogel HJ (2006). Tryptophan- and arginine-rich antimicrobial peptides: structures and mechanisms of action. Biochim. Biophys. Acta 1758: 1184-1202.

Darden T, York D and Pedersen L (1993). Particle mesh ewald: An nlog(n) method for ewald sums in large systems. $J$. Chem. Phys. 98: 10089-10092.

Dutton CJ, Haxell MA, McArthur HAI and Wax RG (2002). Peptide Antibiotics. Discovery, Modes of Action and Applications. Marcel Dekker, New York.

Falla TJ and Hancock RE (1997). Improved activity of a synthetic indolicidin analog. Antimicrob. Agents Chemother. 41: 771-775.

Friedrich CL, Rozek A, Patrzykat A and Hancock RE (2001). Structure and mechanism of action of an indolicidin peptide derivative with improved activity against Gram-positive bacteria. J. Biol. Chem. 276: 24015-24022.

Fuzo CA, Castro JRM and Degrève L (2008). Searching the global minimum of a peptide/bilayer potential energy surface by fast heating and cooling cycles of simulated annealing. Int. J. Quantum Chem. 108: 2403-2407.

Gallivan JP and Dougherty DA (1999). Cation-pi interactions in structural biology. Proc. Natl. Acad. Sci. U. S. A. 96: 9459-9464.

Gallo RL (2005). Antimicrobial Peptides in Human Health Disease. Horizon Bioscience, San Diego.

Gordon YJ, Romanowski EG and McDermott AM (2005). A review of antimicrobial peptides and their therapeutic potential as anti-infective drugs. Curr. Eye Res. 30: 505-515.

Halevy R, Rozek A, Kolusheva S, Hancock RE, et al. (2003). Membrane binding and permeation by indolicidin analogs studied by a biomimetic lipid/polydiacetylene vesicle assay. Peptides 24: 1753-1761.

Hancock RE and Diamond G (2000). The role of cationic antimicrobial peptides in innate host defences. Trends Microbiol. 8: 402-410.

Hess B, Bekker H, Berendsen HJC and Fraaije JGEM (1997). LINCS: A linear constraint solver for molecular simulations. J. Comp. Chem. 18: 1436-1472.

Khandelia $\mathrm{H}$ and Kaznessis YN (2007). Cation- $\pi$ interactions stabilize the structure of the antimicrobial peptide indolicidin near membranes: molecular dynamics simulations. J. Phys. Chem. B 111: 242-250.

Khandelia H, Langham AA and Kaznessis YN (2006). Driving engineering of novel antimicrobial peptides from simulations of peptide-micelle interactions. Biochim. Biophys. Acta 1758: 1224-1234.

Mateo CR, Gómez J, Villalaín J and González-Roz JM (2006). Protein-Lipid Interaction. New Approaches and Emerging Concepts. Springer-Verlag, Berlin.

Mazzé FM, Fuzo CA, Ciancaglini P and Degreve L (2007). Recognition of $\alpha$-helix transmembrane domains with an amphipathy scale generated by molecular dynamics using only the primary sequence of proteins. Genet. Mol. Res. 6: $422-433$.

McPhee JB and Hancock RE (2005). Function and therapeutic potential of host defence peptides. J. Pept. Sci. 11: 677-687.

Miyamoto S and Kollman PA (1992). SETTLE: an analytical version of the SHAKE and RATTLE algorithm for rigid water models. J. Comp. Chem. 13: 952-962.

Murakami MT, Arni RK, Vieira DS, Degreve L, et al. (2005). Correlation of temperature induced conformation change with optimum catalytic activity in the recombinant G/11 xylanase A from Bacillus subtilis strain 168 (1A1). FEBS Lett. 579: 6505-6510.

Powers JP and Hancock RE (2003). The relationship between peptide structure and antibacterial activity. Peptides 24: 1681-1691.

Rozek A, Friedrich CL and Hancock RE (2000). Structure of the bovine antimicrobial peptide indolicidin bound to dodecylphosphocholine and sodium dodecyl sulfate micelles. Biochemistry 39: 15765-15774.

Selsted ME, Novotny MJ, Morris WL, Tang YQ, et al. (1992). Indolicidin, a novel bactericidal tridecapeptide amide from neutrophils. J. Biol. Chem. 267: 4292-4295.

Sitaram N and Nagaraj R (1999). Interaction of antimicrobial peptides with biological and model membranes: structural and charge requirements for activity. Biochim. Biophys. Acta 1462: 29-54.

Subbalakshmi C, Krishnakumari V, Nagaraj R and Sitaram N (1996). Requirements for antibacterial and hemolytic 
activities in the bovine neutrophil derived 13-residue peptide indolicidin. FEBS Lett. 395: 48-52.

Subbalakshmi C, Krishnakumari V, Sitaram N and Nagaraj R (1998). Interaction of indolicidin, a 13-residue peptide rich in tryptophan and proline and its analogues with model membranes. J. Biosci. 23: 9-13.

Tieleman DP, van der Spoel D and Berendsen HJC (2000). Molecular dynamics simulations of dodecyl phosphocholine micelles at three different aggregate sizes: micellar structure and lipid chain relaxation. J. Phys. Chem. B 104: 6380-6388.

van der Spoel D, Lindahl E, Hess B, Groenhof G, et al. (2005a). GROMACS: fast, flexible, and free. J. Comput. Chem. 26: 1701-1718.

van der Spoel D, Lindahl E, Hess B, van Buuren AR, et al. (2005b). GROMACS User Manual version 3.3. Available at http://www.gromacs.org.

Wymore T, Gao XF and Wong TC (1999). Molecular dynamics simulation of the structure and dynamics of a dodecylphosphocholine micelle in aqueous solution. J. Mol. Struct. 485-486: 195-210.

Zasloff M (2002). Antimicrobial peptides of multicellular organisms. Nature 415: 389-395.

Zhang L, Rozek A and Hancock RE (2001). Interaction of cationic antimicrobial peptides with model membranes. J. Biol. Chem. 276: 35714-35722. 